\title{
Inteligencia artificial responsable y ciencia cívica
}

\author{
Responsible artificial intelligence and civic science
}

\author{
ANTONIO LUIS TERRONES RODRÍGUEZ (iDa
}

\section{Resumen}

Tras los procesos de investigación e innovación en el campo de la inteligencia artificial (IA) subyacen relaciones políticas que pueden ser sometidas a una reflexión ética. La ciencia cívica pone en valor la comunicación como una herramienta para fortalecer los vínculos democráticos entre los entornos científicos y la ciudadanía. En este sentido, el propósito de este trabajo gira en torno a la formulación del concepto de inteligencia artificial responsable (IAR) impulsado desde los laboratorios abiertos como un espacio deliberativo para hacer posible el cultivo de habilidades cívicas y democráticas en el contexto tecnológico. Proporcionando de ese modo una orientación pragmática y fronética de los sistemas artificiales hacia los Objetivos de Desarrollo Sostenible. Este trabajo es el resultado de una investigación bibliográfica.

Palabras clave: Inteligencia artificial. Responsabilidad. Ciencia cívica. Laboratorios abiertos. Objetivos de desarrollo sostenible.

\begin{abstract}
Behind the research and innovation processes in the field of artificial intelligence (Al) lie political relationships that can be subjected to ethical reflection. Civic science values communication as a tool to strengthen democratic ties between scientific environments and citizens. In this sense, the purpose of this work revolves around the formulation of the concept of responsible artificial intelligence (IAR) promoted from open laboratories as a deliberative space to make possible the cultivation of civic and democratic skills in the technological context. Thus providing a pragmatic and phonic orientation of artificial
\end{abstract}

\footnotetext{
a Universitat de Valencia, Valencia, España. Máster en Ética y Democracia, e-mail: anluiste@uv.es
} 
systems towards the Sustainable Development Goals. This work is the result of a bibliographic investigation.

Keywords: Artificial intelligence. Accountability. Civic science. Open labs. Sustainable development goals.

\section{Introducción}

La presencia de la inteligencia artificial (IA) en numerosos ámbitos de la vida humana ha suscitado interesantes debates tecnológicos, jurídicos, teológicos, éticos, etc. De igual manera los impactos sociales y políticos se suceden en las sociedades contemporáneas más avanzadas tecnológicamente. Por ello, es importante promover una hermenéutica crítica de las actividades humanas (CORTINA, 1996), con el objetivo de poder impulsar una ética aplicada a la IA que contribuya en la observación de un fenómeno tecnológico que está condicionando la vida de la ciudadanía.

Los modelos de innovación suelen caracterizarse por enfoques monopolistas u oligopolistas que privilegian exclusivamente el criterio de los expertos. Por ello, para promover una democratización del conocimiento científico en el ámbito de la IA, resulta fundamental poner en tela de juicio este modelo y proponer espacios deliberativos desde una conceptualización cívica de la ciencia. La propuesta de una inteligencia artificial responsable (IAR) que permita formular respuestas a las necesidades recogidas en la Agenda 2030 y sitúe al ser humano en la centralidad de sus preocupaciones, es posible llevarla a cabo desde la cultura de una ciencia cívica y los laboratorios abiertos.

Así pues, el propósito de este trabajo consiste en poner en valor las iniciativas europeas en torno a la necesidad de promover la ética en el ámbito de la IA. Subrayando la posibilidad de concretar mecanismos y espacios concretos para poder llevar a cabo esas iniciativas desde las aportaciones de la filosofía moral para el cultivo de habilidades cívicas y el fortalecimiento de la democracia.

\section{Sociedades inclusivas, innovadoras y reflexivas}


Este apartado le debe su título a uno de los retos sociales del Horizonte 2020 (H2020) (COMISIÓN EUROPEA, 2014), Programa Marco de Investigación e Innovación de la Unión Europea (UE), fue presentado en la $7^{\mathrm{a}}$ Conferencia del Programa Marco de I+D+I de la UE en España los días 11 y 12 de noviembre de 2013. Este programa representa importantes oportunidades para la ciencia y la innovación desde diversos sectores, entre los que se encuentran las universidades, centros tecnológicos y de investigación, gobiernos de distintos niveles y el sector empresarial.

Como reza en la página web del H2020 “el objetivo específico de es-te reto social es fomentar una mejor comprensión de Europa, ofrecer soluciones y apoyar una sociedades europeas inclusivas, innovadoras y reflexivas en un contexto de transformaciones sin precedentes y una creciente interdependencia mundial" (COMISIÓN EUROPEA, 2014b). La Comisión Europea (CE) reconoce que las sociedades actuales se enfrentan a enormes retos de carácter socioeconómico que presentan un impacto significativo en diversos ámbitos de creciente interdependencia, entre los que pueden encontrarse: la economía, la cultura, los cambios democráticos, la exclusión y pobreza, las desigualdades, la brecha digital, la pérdida de confianza en las instituciones democráticas, etc. Esta contribución puede contrarrestar los efectos de determinados planes de innovación que han ocasionado un debilitamiento de la inclusión y conciliar de manera sensata y equilibrada la innovación científica con la innovación social, el cultivo de habilidades cívicas y el fortalecimiento de la democracia.

Para construir sociedades inclusivas, innovadoras y reflexivas, es importante poner en valor la educación y la cooperación entre los seres humanos en la búsqueda de soluciones a las problemáticas actuales. La cooperación permite estimular una comunicación intelectual favorecedora de la generación de conocimiento que en los espacios educativos supone un reto decisivo:

La meta de la inteligencia colectiva es el reconocimiento mutuo y el enriquecimiento de las personas. Para ello es necesario que esas personas puedan conversar e interactuar, lo que resulta muy sencillo hoy en día gracias a la tecnología. Internet favorece el intercambio de ideas y conocimientos. Con la Web 2.0 aparecen nuevas formas de relacionarse, en las que los consumidores pasan a ser también creadores, como consecuencia de la facilidad para portar información [...] El cono-cimiento 
absoluto no es posible. Es por esta razón que resulta casi vital la colaboración para «el reconocimiento y el enriquecimiento mutuo de las personas, y no el culto de comunidades fetichizadas o hipostasiadas». El intercambio de conocimiento y experiencias, donde la diferencia es una manera de enriquecer el saber, nos aleja de una uniformada de pensamiento [...] De esta manera, si juntamos todos esos microsaberes, crearemos una inteligencia colectiva, que parte del principio de que cada persona sabe sobre algo. Por tanto nadie tiene el conocimiento absoluto. De lo que resulta fundamental la inclusión y participación de los conocimientos de todos (PARRA; ARENASDOLZ, 2015, p. 123).

En la línea de estas consideraciones políticas por parte de la CE reflejadas en el H2020 fue constituido un grupo de expertos en IA de alto nivel que elaboró el documento Directrices éticas para una IA fiable en 2018. Posteriormente, en enero de 2019 se impulsó el consorcio AI4EU para construir la primera plataforma y ecosistema de una IA con apoyo de la CE en el marco del programa H2020. Un proyecto que se sostiene sobre la idea de la importancia que adquiere compartir recursos de IA producidos en la UE y orientarlos hacia la investigación y la innovación social con un carácter pragmático, y que además facilita la colaboración entre los diversos actores europeos involucrados en el campo de la IA. El diseño de estaba plataforma.

En este contexto de iniciativas políticas por parte de la UE es importante promover en la sociedad una cultura científica de carácter cívico enraizada en una investigación e innovación responsables y en una concepción constructiva tecnología. Así pues, a continuación será presenta-do el concepto de ciencia cívica como una noción alternativa en la posible forma de concebir un vínculo entre lo científico y lo ciudadano.

\section{Ciencia cívica, participación y colaboración ciudadana}

La expresión "ciencia cívica” (civic science) fue empleada por el botánico, sociólogo, científico, urbanista, filósofo, poeta, activista cívico y educador escocés Patrick Geddes (1854-1932), maestro de Lewis Mumfold, gran historiador de la tecnología y de las ciudades. En su obra Ciudades en evolución, escrita en 1915, Geddes (2019) considera que la ciencia cívica consiste en saber que las instituciones y los edificios se levantan desde dentro, sin imposiciones desde arriba ni construcciones de 
afuera. Aboga por un modelo organicista de urbanismo desde una concepción experiencial que pretende establecer unas pautas donde la ciudad y el campo coexistan de forma equilibrada.

El escocés plantea una renovación metodológica, donde la colaboración se lleva a cabo desde diversas perspectivas para promover no solo "construcciones cívicas", sino también el desarrollo de una perspectiva cívica de manera integral, con un método itinerante y participativo. Para ello es importante que estudiantes y futuros planificadores urbanos se sumerjan directamente en la ciudad y practiquen un urbanismo itinerante, centrado no solo en observar la ciudad, sino en escuchar los problemas de los habitantes del lugar y las soluciones que ellos mismos sugieren. Solo de esa forma es posible despertar un auténtico interés por el desarrollo cívico en la ciudadanía.

En el ámbito de la IA el concepto de ciencia cívica aporta una noción de responsabilidad participativa y compartida similar a la que el autor escocés trata de desarrollar en el ámbito del urbanismo. Este concepto, que en este caso pone el acento sobre la dimensión cívica de la ciencia, permite cultivar un espíritu democrático en los procesos de generación de conocimiento científico. El postulado de una IAR sirve para sentar así las bases de una ciencia de carácter cívico que reconozca la necesidad de enriquecer los procesos de generación de conocimiento científico a través de un ejercicio inclusivo, participativo y abierto.

La ciencia cívica promueve la incorporación de aquellos agentes que comúnmente no suelen participar en procesos estrictamente científicos, mediante la deliberación sobre asuntos relativos a la ciencia que presen-tan controversia y que por lo tanto son susceptibles de tratamiento y consideración pública. Contribuye con el proceso de toma de decisiones para que las cuestiones científicas permitan promover la acción cívica y el compromiso ciudadano a través de un diálogo abierto. Valora que los procesos de investigación e innovación sean responsables y para ello sitúa como principal eje articulador la participación abierta e inclusiva de diversos agentes. Este concepto de ciencia cívica ha sido impulsado desde los Estudios Cívicos, un ámbito emergente donde con-fluyen diversas disciplinas que participan en el desarrollo de ideas útiles para la ciudadanía, tejiendo de ese modo un hilo conductor entre teoría y 
práctica. Los Estudios Cívicos surgen como disciplina académica en 2009 en el Jonathan M. Tisch College of Civic Life de la Universidad de Tufts (EE. UU.).

Para Peter Levine (2014) los Estudios Cívicos tratan de responder a la pregunta: «¿Qué debemos hacer?». La importancia de esta pregunta estriba en su carácter de amplitud, de pluralidad, y no de individualidad, pues la resolución de problemáticas, el enfrentamiento de controversias y desafíos, no puede abordarse desde una única perspectiva debido a la complejidad del mundo actual, sino desde una multiplicidad de perspectivas y actuaciones. La Declaración introductoria del Instituto de Verano de Estudios Cívicos señala lo siguiente:

Los Estudios Cívicos representan un campo interdisciplinario enfocado en la reflexión crítica, el pensamiento ético y la acción para el cambio social, dentro y entre las sociedades. Las personas que piensan y actúan juntas para mejorar la sociedad deben abordar los problemas de la acción colectiva (cómo lograr que los miembros trabajen juntos) y la deliberación (cómo razonar juntos sobre los valores en disputa). También deben:

- Comprender cómo se organiza el poder y cómo funciona dentro y entre las sociedades.

- Afrontar los conflictos sociales, la violencia y otros obstáculos a la cooperación pacífica.

- Considerar cuestiones de justicia y equidad cuando surjan tensiones sociales.

- Confrontar las preguntas sobre relaciones apropiadas con personas externas de todo tipo.

- Examinar marcos éticos, políticos y teológicos alternativos para fomentar la reflexión comparativa sobre las diferentes formas en que las personas viven juntas en la sociedad.

El enfoque en la sociedad civil incluye el estudio de la acción colectiva en las esferas sociales que, si bien están organizadas, no pueden ser institucionalizadas ni sancionadas por el Estado. Destaca la perspectiva de los agentes individuales y grupales (School of Arts and Sciences-Tufts University, 2009).

Los Estudios Cívicos se sitúan en la intersección de la reflexión ética, del análisis los hechos y de las estrategias a realizar, conectando teoría y praxis (Kravetz, 
2013: 168). En ese sentido Levine sostiene que son cinco los principios que fundamentan los Estudios Cívicos (2014, p. 30-32):

- Aprender de la deliberación

Los métodos deben caracterizarse por la interacción y la deliberación. No obstante, el procedimiento no es suficiente, pues no se trata de reunir a diversas personas afectadas, sino de promover la discusión desde creencias que permitan interactuar con otros y aprender de ellos, entender que otros conocimientos pueden enriquecer.

- Ser humilde

Ser conscientes de las limitaciones intelectuales que existen a partir de posicionamientos razonablemente cautelosos y humildes con nosotros mismos. En ese sentido, es importante situarse en la senda del pensamiento conservador por un motivo principal, a saber, la resistencia a la arrogancia intelectual, reconociendo la posibilidad de continua mejora.

- Criticar desde dentro

Asumir una posición crítica frente a los problemas y necesidades de la sociedad. Ser conscientes de que en ocasiones pueden surgir contradicciones, pero eso es un símbolo de madurez y complejidad. La crítica no siempre se dirige a grupos humanos en concreto, sino a prácticas y campos.

- Evitar la búsqueda de las causas de raíz

Como seres humanos a través de nuestra experiencia tenemos la capacidad de cambiar elementos estructurales de la realidad sin necesidad de ir a la raíz de los problemas. La raíz de los problemas suele presentar una gran magnitud y eso genera dificultad a la hora de enfocarse en la formulación de alternativas concretas. A veces es mejor abordar un aspecto de un problema concreto, en lugar de atacar un aspecto más fundamental sin éxito.

- Mantener el barco juntos

No limitar el curso de las acciones y las decisiones por un ideal en concreto, sino asumir con conciencia la posibilidad de variar el pensamiento y de buscar nuevos rumbos. En ese sentido, es importante reconocer el valor de la comunidad para esta empresa, entendiendo que el conocimiento se construye de forma colectiva. 
La complejidad e incertidumbre que puede vislumbrarse hoy en la actividad científica hace necesario desarrollar una ciencia cívica para desentrañar esta complejidad y establecer un encuentro con la incertidumbre. Asumiendo que los temas científicos no son exclusivos de los expertos y que pueden ser abordados en la esfera cívica, pues proporciona mayores conocimientos para que las decisiones sean fruto de la buena información obtenida a través de un proceso dialógico entre los actores implicados. Este diálogo sobre la dimensión cívica de la ciencia permite revitalizar la comunidad y poner en valor dinámicas democráticas. La ciencia cívica redefine el concepto de ciencia desde una dimensión pública y se pregunta cómo otros miembros de la comunidad pueden participar en los asuntos científicos complejos. Esta redefinición del concepto de ciencia permite cultivar un enfoque participativo en el entorno de los científicos para fomentar de ese modo la comprensión de la ciudadanía promoviendo la inclusión, lo que Jonathan Garlick y Peter Levine definen como "humanización de la conversación científica" (2016).

En la estela de la ciencia cívica, la Comisión Europea emplea el término "ciencia ciudadana" (citizen science) para referirse al conjunto de niveles de compromiso que pueden desplegarse en el ejercicio científico y que giran en torno a la participación ciudadana.

La ciencia ciudadana abarca una gama de niveles de compromiso: desde estar mejor informado sobre la ciencia hasta participar en el proceso científico mismo mediante la observación, la recopilación o el procesamiento de datos.

La ciencia ciudadana es un término amplio, que abarca la parte de open science en la que los ciudadanos pueden participar en el proceso de investigación científica de diferentes maneras posibles: como observadores, como financiadores, en la identificación de imágenes o el análisis de datos, o al proporcionar datos ellos mismos. Esto permite la democratización de la ciencia, y también está relacionado con el compromiso de los interesados y la participación pública.

Dependiendo de su interés personal, tiempo y recursos tecnológicos, el ciudadano decide cómo participar. Observar avistamientos de aves, identificar galaxias o averiguar cómo plegar proteínas, proporcionando recursos prestando tiempo de computadora o financiamiento directo como en el financiamiento colectivo de proyectos científicos (COMISIÓN EUROPEA, 2017).

Además, la Comisión Europea a través del White Paper on Citizen Science in Europe sostiene que en este tipo de nueva cultura científica se encuentran implícitos los siguientes valores y atributos: 
ATTRIBUTES

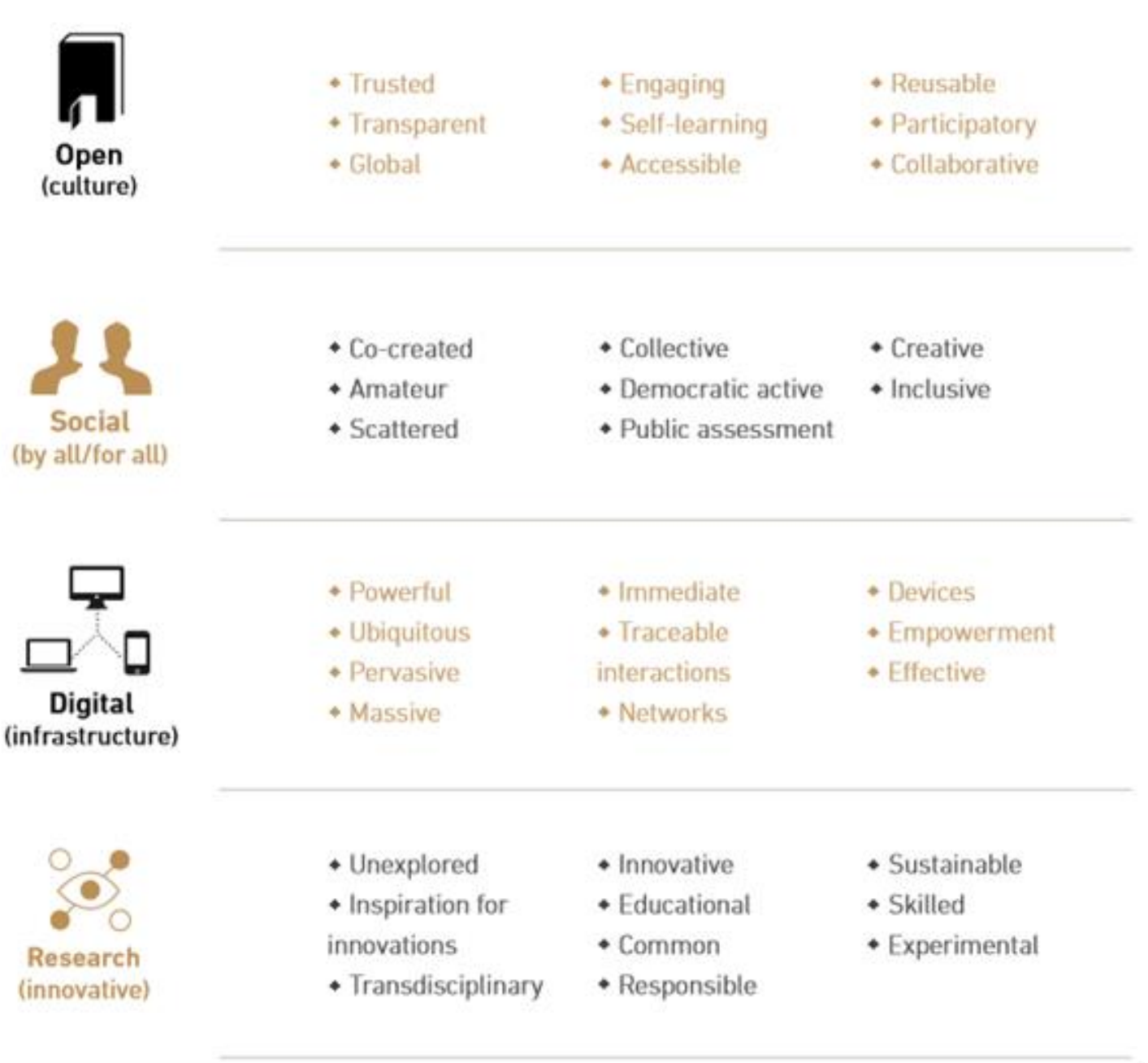

Fuente: Comisión Europea, 2015, p. 10.

La ciencia cívica promueve un cultivo de habilidades comunicativas en el ámbito científico, pues entiende como necesaria la construcción de puentes entre este y otros ámbitos, poniendo en tela de juicio la cultura monopolista y oligopolista. Este cultivo permite fortalecer la sensibilidad y compartir convicciones morales en un ejercicio de diálogo respetuoso. Frente a esa cultura, la ciencia cívica defiende una visión de la ciencia entendida como recurso de interés público, abierto y participativo, susceptible de consideración ciudadana. La humanización de la ciencia conecta con la cultura, la política y los valores a través del diálogo, para que los asuntos científicos no escapen de la comprensión de la ciudadanía.

\section{Antecedentes de la inteligencia artificial responsable}


Las investigaciones sobre la conceptualización de responsabilidad en el ámbito de la IA no son abundantes. Por ello es necesario dirigir la mirada hacia los primeros planteamientos del concepto de IAR. En primer lugar se encuentra el grupo de expertos de alto nivel sobre IA de la Comisión Europea (CE), compuesto por diversas personalidades científicas, entre las que se encuentra Virginia Dignum (2017a; 2017b; 2018), colaboradora en la redacción del documento Directrices éticas para una I A fiable, quién además ha investigado sobre el concepto de IAR. Esta ingeniera sostiene que la responsabilidad debe encontrarse necesariamente vinculada a la inteligencia. Su propuesta de IAR descansa sobre tres pilares fundamentales (2017b, p. 4-5):

- Una preparación previa por parte de la sociedad civil para poder hacer frente a los impactos de la IA. Igualmente los tecnólogos que participen en el diseño e implementación de productos de IA deben estar capacitados en materia de responsabilidad. Este primer pilar está vinculado con el plano educativo y formativo en códigos de conducta. La deliberación entre la ciudadanía y los gobiernos resulta fundamental, aunque esta ingeniera holandesa no ofrece un marco deliberativo concreto.

- Los valores humanos deben estar presentes por medio de modelos y algoritmos en el diseño de los sistemas artificiales. Este es un aspecto que está siendo investigado desde el deep learning.

- Tras el desarrollo de la IA subyacen una serie de relaciones socio-técnicas que presentan un carácter político. En este sentido, es importante promover un espíritu democrático a través de la participación de los agentes involucrados en el desarrollo e impacto de la IA. Este tercer punto resulta fundamental para la posterior formulación que vendrá a hacerse de la IAR en el marco de los laboratorios abiertos. No obstante, Dignum no menciona cuáles son los mecanismos y espacios en los que deben desarrollarse esas relaciones democráticas de participación de los afectados por la IA.

Además, en lo relativo a los principios éticos, Dignum lleva a cabo una contextualización de tres en la actividad de la IA: interactividad-rendición de cuentas, 
adaptabilidad-transparencia y autonomía-responsabilidad (2017 a p. 4698-4699; 2017b, p. 5).

Según Dignum, la deliberación representa un papel fundamental en las tres fases en las que se desarrolla la actividad en el campo de la IA, análisis-diseñoimplementación. Este ejercicio deliberativo ofrecería una aprovechable oportunidad para reflexionar sobre los impactos de diversa índole que se derivan de la IA, que valores se encuentran involucrados en el proceso de diseño tecnológico, y que enfoque moral es el que debe priorizarse mediante mecanismo de adaptabilidad técnica (2017b: 6). Por ello, la responsabilidad adquiriría una dimensión ética y política en el entorno de los intelectos sintéticos, a través del ejercicio de la transparencia. Resulta fundamenta impulsar un cambio cultural en los entornos científicos, para que los expertos tecnólogos entiendan que su actividad no es axiológicamente neutral, pues adquiere implicaciones morales que deberían ser consideradas (2017a, p. 4699).

Otra aportación en materia de responsabilidad en el contexto de la IA es $L a$ Declaración de Montreal para un desarrollo responsable de la IA (2018) que proporciona una serie de orientaciones éticas en el desarrollo de los sistemas artificiales en Canadá. Representa una invitación a la ciudadanía, responsables políticos y personal técnico involucrado en la IA para deliberación en la identificación de once valores éticos: bienestar, autonomía, respecto a la privacidad y la intimidad, solidaridad, participación democrática, equidad, inclusión de la diversidad, prudencia, responsabilidad y desarrollo sostenible. Aunque esta Declaración representa una posible solución ante las problemáticas y controversias derivadas del impacto de la IA, carece de una fundamentación filosófica en profundidad, pues no va más allá de una propuesta deontológica. Es en ese sentido, al igual que la propuesta de Dignum, se trata de un planteamiento débil de IAR debido al insuficiente desarrollo argumentativo y las escasas concreciones sobre un marco deliberativo específico.

Si es admitida la necesidad de una ética aplicada al campo de la IA fundamentada en un método deliberativo que reconozca las complejidades y retos, no cabe duda que tras esa dinámica deliberativa tendrán que surgir decisiones responsables y transparentes. En ese sentido, es fundamental reconocer el trabajo de Dignum y de quienes han elaborado la Declaración de Montreal, aunque estos trabajos podrían enriquecerse concretando un marco deliberativo para la IAR que 
aborde los desafíos desde una ciencia cívica y de un modelo de investigación e innovación abiertas y responsables. Es la filosofía moral la que ofrece bases teóricas y prácticas para orientar una responsabilidad contextualizada en el terreno de la IA e ir más allá de la superficialidad de propuestas débiles. Así pues, a continuación serán presentados los laboratorios abiertos como un espacios deliberativos concretos desde los que promover el proyecto democrático de una IAR.

\section{Laboratorios ciudadanos para la innovación social}

Recogiendo el espíritu de los Estudios Cívicos, y concretamente de la ciencia ć́vica, los laboratorios abiertos son espacios ciudadanos entendidos como un lugar de encuentro para la reflexión colectiva, la generación de conocimiento innovador a partir de propuestas colaborativas y la confluencia de diferentes saberes y sentires. Estos laboratorios se sostienen principalmente sobre dos premisas fundamentales, a saber: la investigación colaborativa y el aprendizaje comunitario en torno a temas de diversa índole. En el contexto concreto de la IA, la motivación para impulsar estos espacios como ecosistemas de innovación colaborativa nace de la posibilidad de cultivar prácticas democráticas y cívicas en el contexto tecnológico a partir de la propuesta de una IAR. Los laboratorios abiertos representan un espacio entendido como una herramienta de innovación ciudadana que contribuirá a propiciar la preparación de las instituciones para las profundas transformaciones que la revolución tecnológica depara.

La cultura que subyace tras un laboratorio abierto sitúa en el centro de su reflexión el concepto de innovación (GARCÍA, 2018, p. 108). La innovación se entiende en términos colectivos, colaborativos y participativos, es decir, desde la inclusión de la ciudadanía como un agente activo ca-paz de organizarse y hacer frente a sus necesidades y anhelos. Un laboratorio abierto sobre ciencia cívica surge de la necesidad de ofrecer lugares públicos de encuentro en los que la ciudadanía pueda reflexionar y contribuir a un discurso científico que impacta directamente sobre sus vidas. 
El valor de los laboratorios cívicos estriba en la cultura comunicativa que se establece en el cultivo de nuevas habilidades de cooperación. Se exploran nuevos mecanismos de intersubjetividad y comunicación para construir colectivamente el conocimiento y mejorar la convivencia en términos cívicos y democráticos a través de la confianza y la colaboración. Estos dos últimos aspectos sirven fundamentalmente para enfrentar la incertidumbre, como sostiene Marcos García: "Los laboratorios ciudadanos ofrecen un lugar para no quedarse en las ideas y prototipar de manera colaborativa anhelos colectivos, y pasar de lo posible a lo realizable" (2018, p. 110).

Existen tres casos de laboratorios cívicos que podrían servir a modo de ejemplo: MedialabPrado, en Madrid; SantaLab, en Santa Fe; y LabIN, en Granada. El primer caso, MedialabPrado, consta de un espacio abierto de encuentro para la producción de proyectos culturales. Cualquier persona, a título individual o colectivo, puede participar de manera colaborativa. Se crean grupos de trabajo y se lanzan convocatorias abiertas para la producción y presentación de proyectos, investigaciones y comunidades de aprendizaje. Entre sus laboratorios se encuentran DataLab, Laboratorio de Datos Abiertos; PrototipaLab, Laboratorio de prototipado creativo; ParticipaLab, Laboratorio de Inteligencia Colectiva para la Participación Democrática; CiCiLab, Laboratorio de Ciencia Ciudadana, etc. En Santa Fe, Argentina, se desarrolla el SantaLAB, un Laboratorio de Innovación Publica que como reza en web «es impulsado como una polí-tica de Innovación y Gobierno Abierto para constituirse en un espacio articulador con las nuevas formas de organización ciudadana y agrupaciones autoorganizadas que, mediante procesos informales de práctica ciudadana, modifican de forma resiliente y adaptativa los entornos que habitamos» (Gobierno de Santa Fe, 2016-2019). SantaLab es una interfaz de colaboración para reunir iniciativas ciudadanas innovadoras sustentadas en el modelo de la cuádruple hélice. El último caso a exponer es el de LabIN, en Granada, el Laboratorio de Innovación Ciudadana de Granada, impulsado por su universidad pública, centrado en la generación de ideas, y también en el prototipado de soluciones y el desarrollo de proyectos para la ciudadanía. Está formado por una red de participación ciudadana y presenta un carácter digital muy notable. 
Concretamente en el ámbito de la IA, existe el caso del Norwegian Open AI Lab, del que se señala lo siguiente en su página web:

\begin{abstract}
El Laboratorio de Inteligencia Artificial Abierto de Noruega es un centro que reúne investigación, educación e innovación dentro de la IA.

Los objetivos de Al Lab son permitir la investigación interdisciplinaria básica y aplicada a un alto nivel internacional, y fomentar asociaciones sólidas entre la academia, el sector privado, el sector público y los institutos de investigación. Brindamos infraestructura y un marco para una estrecha cooperación con diversos socios.

Trabajamos con una variedad de métodos. Hemos seleccionado las siguientes áreas de aplicación clave para nuestra investigación: salud, energía, espacio oceánico, economía digital y entornos inteligentes.

El centro está alojado por el Departamento de Informática de NTNU en Trondheim (https://www.ntnu.edu/ailab).
\end{abstract}

La propuesta del laboratorio abierto está fundamentada en el modelo de Investigación en Innovación Responsables (RRI por sus siglas en inglés, Responsible Research and Innovation) que pone en valor la participación de los grupos de interés en el ejercicio deliberativo que debe estar presentes en el diseño de los sistemas con inteligencia artificial. Este modelo puede definirse de la siguiente manera:

\begin{abstract}
Un proceso transparente e interactivo mediante el cual los actores sociales y los innovadores se hacen mutuamente sensibles entre sí en vista de la aceptabilidad (ética), la sostenibilidad y la conveniencia social del proceso de innovación y sus productos comercializables (con el fin de permitir una integración adecuada de los avances tecnológicos en nuestra sociedad) [...] La innovación responsable significa cuidar el futuro. A través de la administración colectiva de la ciencia y la innovación en el presente (VON SCHOMBERG, 2011, p. 41).
\end{abstract}

En el terreno de la ética la deliberación se convierte una potente herramienta para el abordaje de determinas problemáticas y controversias, y como Tomás Domingo Moratalla y Lydia Feyto Grande afirman: «la deliberación es el método de análisis ético una vez que reconocemos la contingencia de los asuntos humanos y comprendemos que nuestro ámbito de acción y decisión es el de la incertidumbre y la complejidad» (2017, p. 41). Una deliberación inclusiva que representa el elemento central que el laboratorio abierto obtiene de este modelo, ya que responde a un interés social por una investigación e innovación exigentes en términos de responsabilidad inclusiva con todos los actores implicados en la actividad mediante la confianza dialógica (FERNÁNDEZ-BELTRÁN et al., 2017, p. 1044). Para establecer una 
comparativa aclaratoria, es importante mencionar que estos grupos son denominados en el ámbito de la ética empresarial y Responsabilidad Social Corporativa (RSC) como stakeholders (GONZÁLEZ ESTEBAN, 2007, p. 208-209). El modelo RRI articula una adaptabilidad práctica en los laboratorios abiertos y enriquece los procesos informativos y anticipativos para que las perspectivas que aportan los grupos de interés adquieran relevancia en el diseño de la IA. El modelo RRI se ilustra de la siguiente manera:

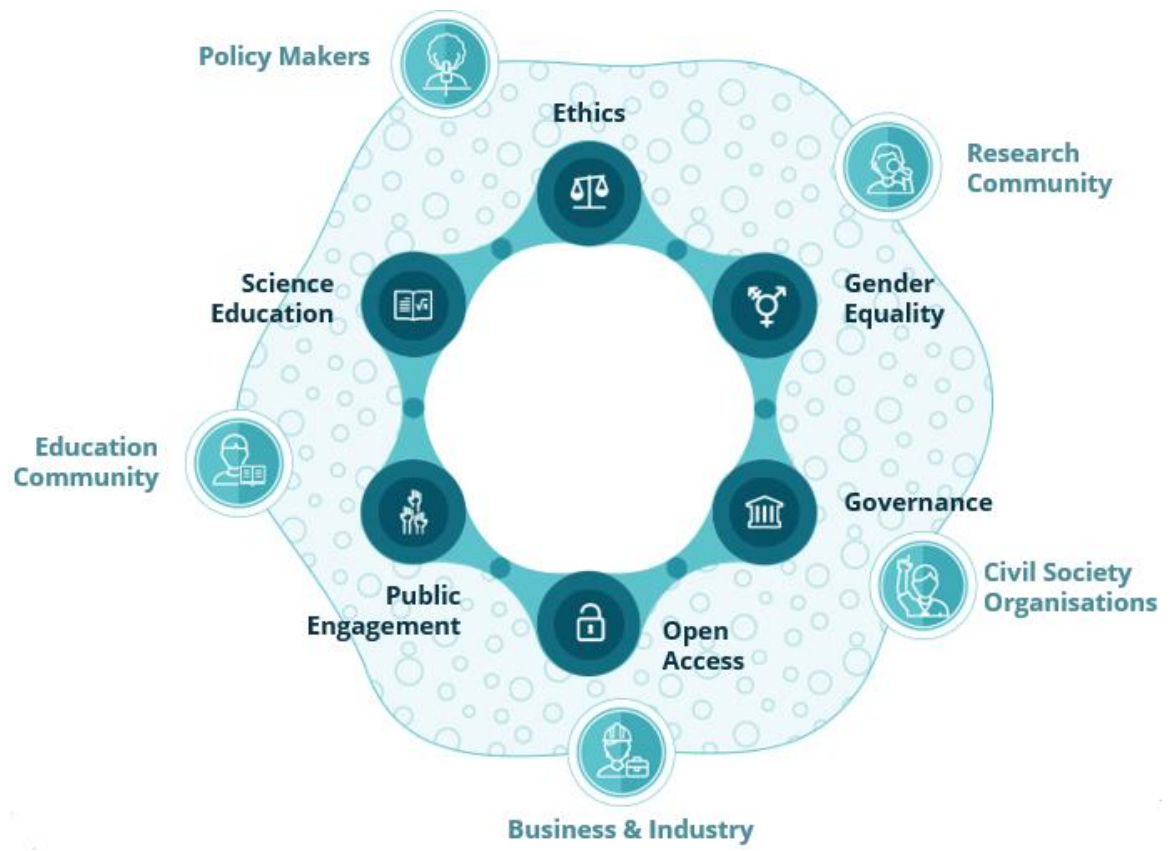

Fuente: https://www.rri-tools.eu/es.

En el laboratorio abierto sobre IA se encuentra presente una concepción constructiva de la evaluación tecnológica (MUÑOZ-ALONSO LÓPEZ, 1997) que observa la necesidad de cultivar una dimensión relacional en el contexto tecnológico para promover una noción compartida del mundo. Poniendo de ese modo en tela de juicio los enfoques monopolistas y oligopolistas en los procesos de innovación, propios de la triple hélice (Urra Canales, 2018, p. 184):

[...] la relacionalidad lleva a la humanidad a ser construida no solo por los acto-res, sino que también reconoce que estos actores se construyen a través de sus relaciones con los demás [...] este proceso no consiste en lograr una única respuesta autorizada y absoluta, sino de eliminar soluciones problemáticas y buscar opciones creativas 
surgidas mediante una comparación entre diferentes posibilidades (RO-YAKKERS; TOPOLSKI, 2014, p. 355-356).

En el caso de la IA, un laboratorio abierto sobre ciencia cívica podría contribuir favorablemente en la promoción de una IAR, respondiendo a las necesidades de la ciudadanía y cultivando habilidades cívicas y democráticas en el ámbito tecnológico. Visto así, sería un espacio de confluencia de saberes para la búsqueda de soluciones en términos pragmáticos, con la participación de los actores implicados en el despliegue de la actividad de la IA aportando una mirada sobre el impacto de esta tecnología. Todo ello contribuiría a una innovación científica abierta, comprometida con la defensa de los derechos humanos y la promoción de los Objetivos de Desarrollo Sostenible (ODS) (ORGANIZACIÓN DE NACIONES UNIDAS, 2015), situando al ser humano en la centralidad de los procesos de investigación e innovación.

\section{Bienestar humano y Objetivos de Desarrollo Sostenible}

¿Es posible situar la riqueza y el valor del ser humano en la centralidad de las motivaciones de los proyectos en IA? Los importantes desafíos que enfrentan las sociedades de este tiempo, exigen un desarrollo cien-ífico que permita dar respuesta a las necesidades de la humanidad reconocidas por los ODS. El documento elaborado por los 52 expertos de alto nivel de la Comisión Europea bajo el título Directrices éticas para una IA fiable, y anteriormente mencionado, señala lo siguiente:

[...] La IA no es un fin en sí mismo, sino un medio prometedor para favorecer la prosperidad humana y, de ese modo, mejorar el bienestar individual y social y el bien común, además de traer consigo progreso e innovación. En particular, los sistemas de IA pueden ayudar a facilitar el logro de los Objetivos de Desarrollo Sostenible de las Naciones Unidas, como la promoción del equilibrio entre mujeres y hombres y la lucha contra el cambio climático, la racionalización del uso que los seres humanos hacemos de los recursos naturales, la mejora de la salud, la movilidad y los procesos de producción y el seguimiento de los avances en los indicadores de sostenibilidad y cohesión social.

10) Para ello, es necesario que los sistemas de IA se centren en las personas y se fundamenten en el compromiso de utilizarlos al servicio de la humanidad y del bien común, con el objetivo de mejorar el bienestar y la libertad de los seres humanos (COMISIÓN EUROPEA, 2019, p. 5). 
Esta reivindicación de la centralidad humana en los procesos de generación de conocimiento en el entorno de la IA, forma parte de un humanismo tecnológico fundamentado en un pensamiento pragmático y fronético. En términos políticos la IA podría beneficiar al ser humano desde una noción de utilidad que forma parte de la propia condición técnica de él, como John Dewey enfatizó en 1930:

'Tecnología' significa todas las técnicas inteligentes por las que las energías de la naturaleza y del hombre son dirigidas y utilizadas en la satisfacción de las necesidades humanas, no se puede limitar a unas pocas, exteriores y relativamente mecánicas formas. A la vista de sus posibilidades, la concepción tradicional de experiencias es obsoleta (DEWEY, 1984, p. 277).

No obstante, es importante destacar que en la propuesta de una ciencia cívica formulada desde una IAR, este fundamento pragmático se encontraría sustentado en criterios éticos deliberados y razonables.

De igual manera ha sido mencionado el pensamiento fronético como el sustento fundamental de un humanismo tecnológico que da forma a la propuesta esbozada en este trabajo de orientación de los intelectos sintéticos en beneficio de las personas. Este pensamiento señala que más allá de los aspectos estrictamente científicos y técnicos, se da lugar la implicación de una serie de valores que influyen en los juicios y decisiones a la hora de dirigir la mirada hacia la investigación y la innovación (FLYVBJERG, 2001; 2004; 2006a; 2006b). Bent Flyvbjerg señala la posibilidad de establecer un entrelazamiento de los saberes de las humanidades y las ciencias sociales, a fin de caminar más allá de una férrea cultura epistémica inspirada en las ciencias naturales. Buscando situarse de ese modo en el espacio de lo fronético, entendido como un pensar más amplio e integral, mediante el reconocimiento de otras variables y notas características de la realidad que la tecnocracia mutila u omite.

Para situar el valor humano en la centralidad de los diseños de IA en un contexto democrático, es importante comenzar reconociendo la presencia de una imperante tecnocracia que coloniza diversas esferas de la vida y las instituciones. La oleada tecnocrática se ha venido instaurando desde la segunda mitad del siglo XX en las sociedades actuales, donde lo técnico ha despuntado. Jordi Pigem define la tecnocracia de la siguiente manera: 
En los últimos treinta años la democracia ha ido siendo desplazada por la tecnocracia. La tecnocracia, como su nombre indica, es el control de la economía y de la sociedad a partir de criterios no humanos, sino exclusivamente técnicos. Tal como un ordenador funciona aplicando algoritmos (secuencias de reglas y cálculos), la tecnocracia solo atiende a modelos abstractos y secuencias de fórmulas y estadísticas. [...] La tecnocracia presume de eficiencia. A corto plazo y en ámbitos estrictamente cuantificables, parece obtenerla. A largo plazo y desde una perspectiva más amplia, vemos que no. Al reducirlo todo a abstracciones, pierde de vista el mundo real y en vez de eficiencia genera negligencia (PIGEM, 2013, p. 16 y 25-26)

El paradigma tecnocrático, de raíz cientificista, identifica que la solución de los problemas que padece la humanidad como consecuencia del poder de la técnica, estriban en ella misma, sin necesidad de recurrir a otros saberes alternativos. En cambio, como sostiene el Papa Francisco en la encíclica Laudato si', las soluciones a los problemas actuales no pueden ser meramente técnicas:

Buscar solo un remedio técnico a cada problema ambiental que surja es aislar cosas que en la realidad están entrelazadas y esconder los verdaderos y más profundos problemas del sistema mundial (2015, p. 35).

Cualquier solución técnica que pretendan aportar las ciencias será impotente para resolver los graves problemas del mundo si la humanidad pierde su rumbo $(2015, \mathrm{p}$. 62).

Las soluciones meramente técnicas corren el riesgo de atender a síntomas que no responden a las problemáticas más profundas (2015, p. 46).

Resulta sensato afirmar que es pertinente priorizar la orientación de la investigación y la innovación del campo de la IA hacia los ODS como una respuesta alternativa a la tecnocracia imperante para poner en valor y proteger a la humanidad. Así pues, la importancia concedida a los ODS subraya la posibilidad de promover habilidades cívicas y fortalecimiento democrático desde los procesos de generación de conocimiento en el entorno de la tecnología e impulsar una IAR.

\section{Conclusión}

Como ha podido comprobarse a lo largo de las páginas que anteceden, tras los procesos de generación de conocimiento científico en el campo de la IA subyacen una serie de relaciones políticas que pueden ser sometidas a una reflexión ética. 
En la línea de un pensamiento democrático fundamentado en la deliberación como una herramienta útil para hacer frente a la resolución de problemáticas de diversa índole, es importante dirigir la mirada hacia en el entorno de los intelectos sintéticos. En este sentido, se ha plantea-do la formulación del concepto de IAR como una respuesta pragmática desde la que poner en valor la participación de la ciudadanía en los procesos de investigación e innovación científica. No obstante, esta formulación va precedida de la necesidad de un cambio cultural en los entornos científicos que debería ir de la mano de una ciencia cívica.

La idea del laboratorio abierto sobre IAR como un espacio colaborativo representa una oportunidad para la innovación social en aquellos asuntos que afectan a la ciudadanía y en la que puede llevarse a cabo un ejercicio para valorar la construcción de un hilo conductor entre la aceptabilidad y la deseabilidad de los procesos de generación de conocimiento, a través de una ética aplicada fundamentada en el diálogo. No obstante, antes de finalizar, es importante reconocer que el encuentro transdisciplinar en los laboratorios abiertos debería ir de la mano de una integración de las humanidades con los saberes científicos y tecnológicos. Que tendría que comenzar a partir de una educación STEAM (siglas en inglés de Sciencie, Technology, Engineergin, Arts and Math) (YAKMAN, 2010; 2018). Experiencias como el proyecto AI4EU de la Unión Europea, significan los primeros pasos para la democratización del conocimiento en el ámbito de la IA y la creación de un ecosistema tecnológico más cívico.

\section{Bibliografía}

COMISIÓN EUROPEA. Horizon 2020. El Programa Marco de Investigación e Innovación de la Unión Europea. Luxemburgo: Oficina de Publicaciones de la Unión Europea, 2014. Disponible en:

https://ec.europa.eu/programmes/horizon2020/sites/horizon2020/files/H2020_ES_KI0 213413ESN.pdf. Acceso en: 8 mayo 2020.

COMISIÓN EUROPEA. White Paper on Citizen Science in Europe. 2015. Disponible en: https://ec.europa.eu/futurium/en/system/files/ged/socientize_white_paper_on_citizen_s cience.pdf. Acceso en: 8 de mayo de 2020.

COMISIÓN EUROPE. Citizen Science. Shaping Europe's digital future, 2017. Disponible en: https:/ / ec.europa.eu/digital-single-market/en/citizen-science. Acceso en: 8 de mayo 2020. 
COMISIÓN EUROPEA. Directrices éticas para una IA fiable, 2019. Disponible en: https://ec.europa.eu/digital-single-market/en/news/ethics-guidelines-trustworthy-ai.

Acceso en: 8 mayo 2020.

CORTINA ORTS, A. El estatuto de la ética aplicada. Hermenéutica crítica de las actividades humanas. Isegoría. Revista de filosofía moral y política, v. 13, p. 119-134, 1996.

DEWEY, J. La experiencia y la naturaleza. México: Fondo de Cultura Económica, 1948.

DIGNUM, V. Responsible Autonomy. In: International Joint Conference on Artificial Intelligence, 2017a.

DIGNUM, V. Responsible artificial intelligence: designing AI for human values. ITU Journal ICT Discoveries, v. 1, 2017b.

DIGNUM, V. Ethics in artificial intelligence: introduction to the special issuse. Ethics and Information Technology, v. 20, p. 1-3, 2018.

DOMINGO MORATALLA, T.; FEITO GRANDE, L. Bioética narrativa: clave de la deliberación. En: LEÓN CORREA, F. J.; SOROKIN, P. (Coord.). Bioética y salud pública en y para América Latina. Santiago de Chile: Federación Latinoamericana y del Caribe de Instituciones de Bioética, 2017. p. 37-59.

FERNÁNDEZ-BELTRÁN, F.; GARCÍA-MARZÁ, D.; SANAHUJA, R.; ANDRÉS MARTÍNEZ, A.; BARBERÁ FORCADELL, S. La gestión de la comunicación para el impulso de la Investigación e Innovación Responsables: propuesta de protocolo desde la ética dialógica. Revista Latina de Comunicación Social, v. 72, p. 1040-1062, 2017. Disponible en: http://www.revistalatinacs.org/072paper/1207/57es.html. Acceso en: 8 mayo 2020.

FRANCISCO, S. P. Laudato si'. 2015. Disponible en: http://www.vatican.va/content/francesco/es/encyclicals/documents/papafrancesco_20150524_enciclica-laudato-si.html. Acceso en: 8 de mayo 2020.

FLYVBJERG, B. Making Social Science Matter: Why Social Inquiry Fails and How It Can Succeed Again. Cambridge: Cambridge University Press, 2001.

FLYVBJERG, B. Cinco malentendidos acerca de la investigación mediante los estudios de caso. Revista Española de Investigaciones Sociológicas, v. 106, n. 4, p. 33-62, 2004.

FLYVBJERG, B. Social Science That Matters. Foresight Europe, v. 2, p. 38-42, 2006 a.

FLYVBJERG, B. Making Organization Research Matter: Power, Values, and Phronesis. En: CLEGG, S. R.; HARDY, C.; LAWRENCE, T. B. Y; NORD, W. R. (Eds.). The Sage Handbook of Organization Studies. CA: Thousand Oaks, 2006b. p. 370-387.

GARCÍA, M. Los laboratorios ciudadanos en los sistemas de experimentación e innovación. En: VV.AA. Abrir instituciones desde dentro. Aragón: Laboratorio de Aragón Gobierno Abierto, 2018. Disponible en: http://www.laaab.es/hackinginside/. Acceso en: 8 mayo 2020.

GARLICK, J. Y LEVINE, P. Where civics meets science: Building science for the public good through Civic Science. Oral Diseases, v. 23, n. 6, 2016.

GEDDES, P. Ciudades en evolución. Oviedo: KRK, 2009. 
GOBIERNO DE SANTA FE. SantaLab: Laboratorio de Innovación Pública. Provincia de

Santa Fe: Modernización del Estado, 2016-2019. Disponible en: https://www.santafe.gob.ar/index.php/web/content/view/full/203591/(subtema)/93686. Acceso en: 8 de mayo de 2020 .

González Esteban, E. La teoría de los stakeholders. Un puente para el desarrollo práctico de la ética empresarial y de la responsabilidad social corporativa. Veritas, 17 (2), p. 205-224. 2007.

KRAVETZ, K. Civic Studies: Bringing Theory to Practice. The Good Society, vv. 22, n. 2, p. 162-171, 2013.

MUÑOZ-ALONSO LÓPEZ, G. La evaluación de tecnologías (ET): origen y desarrollo. Revista General de Información y Documentación, v. 7, n. 1, p. 15-30, 1997.

LEVINE, P. Civic Studies. Philosophy \& Public Policy Quarterly, v. 31, n. 1, p. 29-33, 2014.

ORGANIZACIÓN DE NACIONES UNIDAS. Objetivos de Desarrollo Sostenible, 2015. Disponible en: https://www.undp.org/content/undp/es/home/sustainable-developmentgoals.html. Acceso en: 8 mayo 2020.

PARRA, N.-H.; ARENAS-DOLZ, F. Revolución tecnológica y democracia del conocimiento. Por una universidad innovadora. Valencia: Laboratorio de la Sociedad del conocimiento, 2015.

PIGEM, J. La nueva realidad. Barcelona: Kairós, 2013.

ROYAKKERS, L. Y TOPOLSKI, A. Military Robotics \& Relationality: Criteria for Ethical Decision-Making. En: VAN DEN HOVEN, J. et al (Eds.). Responsible Innovation 1: Innovative Solutions for Global Issues. New York: Springer, 2014. p. 351-368.

RUÍZ MARCOS, L. Experimentar en las instituciones culturales: el ejemplo de los laboratorios ciudadanos. Laboratorio de Aragón Gobierno Abierto, 2018. Disponible en: http:/ /www.laaab.es/2018/07/experimentar-en-las-instituciones-culturales-el-ejemplo-delos-laboratorios-ciudadanos/. Acceso en: 8 mayo 2020.

SCHOOL OF ARTS AND SCIENCES. Tufts University. Civic Studies, 2009. Disponible en: https://as.tufts.edu/civicstudies/about. Acceso en: 8 mayo 2020.

UNIVERSITE DE MONTREAL. Montreal Declaration for responsible AI development, 2018. Disponible en:

https://docs.wixstatic.com/ugd/ebc3a3_c5c1c196fc164756afb92466c081d7ae.pdf. Acceso en: 8 mayo 2020 .

URRA CANALES, M. Estado, mercado, academia... y comunidad. Una cuádruple hélice para el desarrollo integral y la innovación (tesis doctoral). Madrid: Universidad Pontificia Comillas, 2017.

VON SCHOMBERG, R. Prospects for technology assessment in a framework of responsible research and innovation. En: DUSSELDORP, M.; BEECROFT, R. (Eds). Technikfolgen abschätzen lehren: Bildungspotenziale transdisziplinärer Methoden (3961).Wiesbaden: Vs Verlag, 2011. p. 39-61. 
YAKMAN, G. What is the point of STE@M?-A Brief Overview, 2010. Disponible en: https://www.researchgate.net/publication/327449281_What_is_the_point_of_STEAMA_Brief_Overview. Acceso en: 8 mayo 2020.

YAKMAN, G. STEAM Pyramid History, 2018. Disponible en: https://steamedu.com/pyramidhistory/. Acceso en: 8 mayo 2020.

RECIBIDO: 08/05/2020

RECEIVED: 05/08/2020

APROBADO: 01/10/2020

APPROVED: $10 / 01 / 2020$ 